\title{
COMPORTAMENTO TÉRMICO DE AMBIENTES INTERNOS SOB A INFLUÊNCIA DE ENVOLTÓRIAS VERDES
}

\section{THERMAL BEHAVIOUR OF INDOOR SPACES UNDER THE INFLUENCE OF GREEN ENVELOPES}

\author{
Ketlin Bruna Montanari 1 \\ Universidade Estadual de Campinas, Campinas, SP, Brasil, kmontanari@gmail.com \\ Lucila Chebel Labaki 2 \\ Universidade Estadual de Campinas, Campinas, SP, Brasil, lucila@fec.unicamp.br
}

\begin{abstract}
Resumo
As envoltórias verdes são um meio de tornar a vegetação parte integrante da edificação e de seus sistemas, tais como o de resfriamento passivo. No entanto, as dinâmicas que sustentam o bom desempenho térmico destas envoltórias dependem de diversas variáveis. O objetivo deste artigo foi identificar, através de revisão sistemática da literatura recente sobre o tema, o comportamento térmico de ambientes construídos internos sob a influência das envoltórias verdes e verificar quais são as possíveis variáveis de desempenho térmico, conforme suas características. Os trabalhos analisados mostram que a redução de temperatura do ambiente interno é significativa, sendo mais acentuada no caso de paredes verdes. O desempenho térmico das envoltórias verdes depende de variáveis como o tipo de vegetação, densidade de folhagem, composição do substrato, etc., destacando as características climáticas, apesar da maioria das pesquisas focar em resultados de outras variáveis. Foi possível, também, verificar pontos frágeis do conhecimento atual, como as lacunas a respeito do peso da umidade do substrato no resfriamento de coberturas verdes e da falta de estudos que comparem o desempenho de coberturas verdes com coberturas de telhas cerâmicas ou de fibrocimento. A análise das variáveis identificou que estas exercem entre si relações multifacetadas, o que tornou evidente que determinar hierarquias entre estas não é possível e nem prudente. A análise da coerência entre as variáveis é mais importante do que a determinação de hierarquia entre as mesmas, bem como esta ação deve preceder a definição das características de uma determinada envoltória verde a ser implantada em dado lugar.
\end{abstract}

Palavras-chave: Cobertura verde. Parede verde. Fachada verde. Comportamento térmico. Variáveis de desempenho.

\begin{abstract}
Green envelopes are a means for vegetation to become an integral part of the building and its systems such as the passive cooling. However, the dynamics that sustain a good thermal performance of these envelopes depend on many variables. This article aimed to identify, through the systematic review of recent literature about the topic, the thermal behavior of internal built environments under the influence of green envelopes and which are the possible performance variables according to its features. The analyzed papers show that the temperature reduction of indoor spaces is significant, being more pronounced in the case of green walls. The thermal performance of green envelopes depends on variables such as the vegetation type, leaf density, substrate's composition, etc., highlighting the climatic features despite the fact that most studies focus on results from other variables. It was also possible to verify weak spots of the current knowledge, like the gaps regarding the weight of the substrate's humidity in green roofs' cooling and the lack of investigations that compare the green roof's performance with ceramic tile or fibro cement roofs. The analysis of the variables identified that these exert multifaceted relations between themselves, which made evident that settling hierarchies between these are neither possible nor prudent. The study of the coherence between the variables is more important than determining its hierarchy, as well as this action, must precede the definition of characteristics of a given green envelope to be installed in a specific place.
\end{abstract}

Keywords: Green roof. Green wall. Green façade. Thermal behaviour. Performance variables.

How to cite this article:

MONTANARI, Ketlin Bruna; LABAKI, Lucila Chebel. Comportamento térmico de ambientes internos sob a influência de envoltórias verdes. PARC Pesquisa em Arquitetura e Construção, Campinas, SP, v. 8, n. 3, p. 181-193, set. 2017. ISSN 1980-6809. Disponível em:

<https://periodicos.sbu.unicamp.br/ojs/index.php/parc/article/view/8650241>. Acesso em: 20 jan. 2018.

doi:http://dx.doi.org/10.20396/parc.v8i3.8650241 


\section{Introdução}

A vegetação é uma estratégia de relevância na prática da arquitetura bioclimática, por proporcionar melhoria do conforto térmico e, consequentemente, economia de energia. As envoltórias vegetadas (verdes), que podem ser coberturas, paredes ou fachadas verdes (PERINI; ROSASCO, 2016), são instalações que podem tornar a vegetação parte integrante da edificação e de seus sistemas, proporcionando benefícios como o resfriamento passivo.

Os benefícios proporcionados pelas envoltórias verdes, dependendo da escala de sua implantação (em escala urbana ou da edificação) podem contribuir com melhoria da qualidade do ar (LUO et al., 2015; MARCHI et al., 2015), amortecimento térmico de superfícies construídas (YANG et al., 2015), redução de reflexo luminoso de edificações nas ruas e no interior das próprias edificações (VIRK et al., 2015), retenção de água pluvial (HARPER et al., 2015; SEBTI; BENNIS; FUAMBA, 2016; VERSINI et al., 2015), redução de metais pesados e acidez da água pluvial (SHAFIQUE; KIM, 2015; VIJAYARAGHAVAN; JOSHI, 2014), aumento da biodiversidade (LIN; PHILPOTT; JHA, 2015; MADRE et al., 2014; MULLER et al., 2014), conforto acústico (AZKORRA et al., 2015; CONNELLY; HODGSON, 2015), bem-estar psicológico (ABDUL-RAHMAN et al., 2014; SCHERER; FEDRIZZI, 2014) e economia de energia por reduzir a necessidade de uso do ar condicionado para resfriamento (COMA et al., 2014; LA ROCHE; BERARDI, 2014; WONG; BALDWIN, 2016) e aquecimento (CAMERON; TAYLOR; EMMETT, 2014; LUNDHOLM; WEDDLE; MACIVOR, 2014).

O desempenho térmico das envoltórias verdes se estende para além dos benefícios voltados às temperaturas internas da edificação. Conforme Ambrosini et al. (2014) e Santamouris (2014), entre diversas outras pesquisas, aponta-se como benefícios térmicos externos das envoltórias verdes o auxílio na moderação do clima e na redução de ilhas de calor urbanas. No entanto, as dinâmicas que sustentam o bom desempenho térmico destas envoltórias, tanto nas temperaturas internas quanto externas, dependem de diversas variáveis, como temperatura do ar, umidade relativa, velocidade do vento, radiação solar, entre outras (MOODY; SAILOR, 2013; PENG; JIM, 2015).

Em atenção a este fato, o objetivo deste artigo foi identificar o comportamento térmico de ambientes internos construídos sob a influência das envoltórias verdes e quais as possíveis variáveis de desempenho térmico, conforme suas características, através de revisão sistemática da literatura recente sobre o tema, nacional e internacional. As temperaturas externas à edificação não foram observadas nesta pesquisa, devido ao fato de que a complexidade e a extensão do assunto impossibilitam o detalhamento necessário à compreensão em um único artigo.

\section{Método}

O método de revisão sistemática da literatura (BRERETON et al., 2007; ENGSTRÖM; RUNESON, 2011) foi aplicado, o que resultou em um panorama dos estudos científicos disponíveis sobre o tema e sua distribuição ao longo dos anos, bem como métricas a respeito dos artigos em português e inglês aderentes ao tema e os resultados acerca do foco da pesquisa. Os assuntos explorados para atender ao objetivo da pesquisa consistiram em redução média de temperatura das envoltórias verdes, variáveis de desempenho térmico das envoltórias verdes e relação destas com a economia de energia.

A revisão sistemática da literatura consiste, de acordo com Brereton et al. (2007), em cinco passos, que se resumem em transformar a necessidade de informação em uma questão a ser respondida, encontrar as melhores evidências para responder a questão e avaliar criticamente as evidências em relação à aderência à questão, validade e impacto.

\section{Definição do foco}

Dentro do assunto de desempenho térmico do ambiente construído, o tema desta pesquisa foi o desempenho térmico de ambientes internos sob a influência de envoltórias verdes, com foco nesta influência e em identificar as possíveis variáveis de desempenho térmico conforme as características da envoltória vegetada em questão. Desta forma, a questão é: qual é a influência que as envoltórias vegetadas desempenham na temperatura interna das edificações e quais são as variáveis de desempenho?

\section{Busca dos termos}

A partir do tema, foi definido o string de busca. Todos os termos possíveis para envoltórias vegetadas em inglês, que já eram conhecidos através de pesquisa prévia de revisão bibliográfica, foram utilizados. Para focar no desempenho térmico interno das edificações, termos como thermal performance, thermal comfort e variações similares foram utilizados, excluindo grande parte da questão térmica externa com a expressão "NOT 'outdoor thermal" "acrescida ao final do string.

As bases de dados internacionais utilizadas foram Web of Science e Compendex. Para encontrar pesquisas brasileiras, os termos em português equivalentes aos do string de busca em inglês foram buscados na base Scielo, sem adicionar os termos que filtrassem apenas os de desempenho térmico, devido ao número muito reduzido 
de artigos em português existentes sobre envoltórias verdes em geral.

As palavras-chave resultantes, bem como a pesquisa destas e os filtros aplicados estão descritos na Tabela 1. A classificação das pesquisas aderentes de acordo com a base de dados é descrita na Figura 1.

Tabela 1 - Palavras-chave da pesquisa e detalhamento da filtragem

\begin{tabular}{|c|c|c|}
\hline \multicolumn{2}{|c|}{$\begin{array}{c}\text { Base de dados } \\
\text { (Web of Science, Compendex e Scielo) }\end{array}$} & $\begin{array}{c}\text { Trabalhos } \\
\text { encontrados }\end{array}$ \\
\hline $\begin{array}{l}\text { Palavras- } \\
\text { chave }\end{array}$ & $\begin{array}{l}\text { ("green façade*" or "green wall"” or } \\
\text { "vertical garden*" or "vertical greenery } \\
\text { system*" or "vegetated wall*" or "living } \\
\text { wall*" or "green terrace*" or "green } \\
\text { roof*" or "rooftop garden*" or "building } \\
\text { greenery system*" or "greenery } \\
\text { cover*" or "green envelope*" or } \\
\text { "vegetated roof*" or "vegetation roof"” } \\
\text { or "vegetated envelope*" or "vertical } \\
\text { greening" or "vegetative coverage" or } \\
\text { "vegetal façade" or "facade greening") } \\
\text { and ("thermal performance" or } \\
\text { "building comfort" or "thermal effect" } \\
\text { or "thermal insulation" or "thermal } \\
\text { comfort") not "outdoor thermal" }\end{array}$ & 397 \\
\hline \multirow{7}{*}{ Filtros } & Apenas artigos & 325 \\
\hline & Apenas artigos em português e inglês & 313 \\
\hline & Apenas artigos de periódicos & 265 \\
\hline & $\begin{array}{c}\text { Apenas artigos de periódicos dos } \\
\text { últimos cinco anos }\end{array}$ & 192 \\
\hline & Excluindo repetições & 153 \\
\hline & Análise dos títulos & 97 \\
\hline & Leitura exploratória dos artigos & 59 \\
\hline \multicolumn{2}{|c|}{ Total de pesquisas aderentes } & 59 \\
\hline
\end{tabular}

${ }^{\star}$ Na base Scielo foram buscados apenas termos em português

Fonte: As autoras.

No filtro "apenas artigos de periódicos", antes de restringir para os últimos cinco anos, os artigos foram classificados por ano de publicação, conforme Figura 2. Analisando essa classificação, foi percebida a atualidade do tema, com resultados não anteriores ao ano de 2001 (Figura 2). O ano de 2017 aparece com poucos resultados por conta da pesquisa englobar artigos publicados até o dia 3 de fevereiro de 2017, data da pesquisa nas bases de dados. Os últimos cinco anos foram selecionados tendo em vista a análise da bibliografia mais recente sobre o tema.

Figura 1 - Pesquisas aderentes de acordo com a base de dados

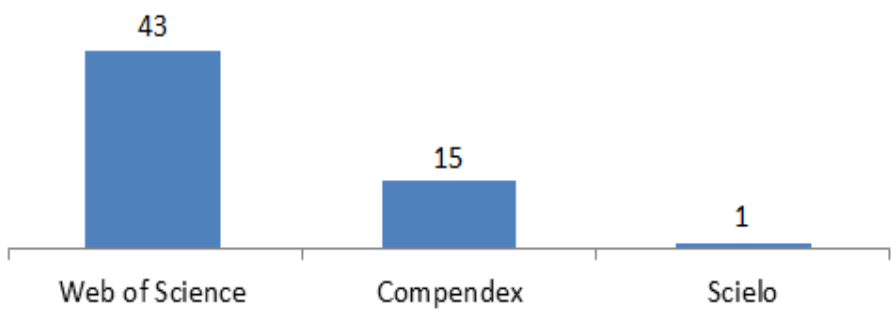

Fonte: As autoras.
Figura 2 - Artigos de periódicos por ano de publicação

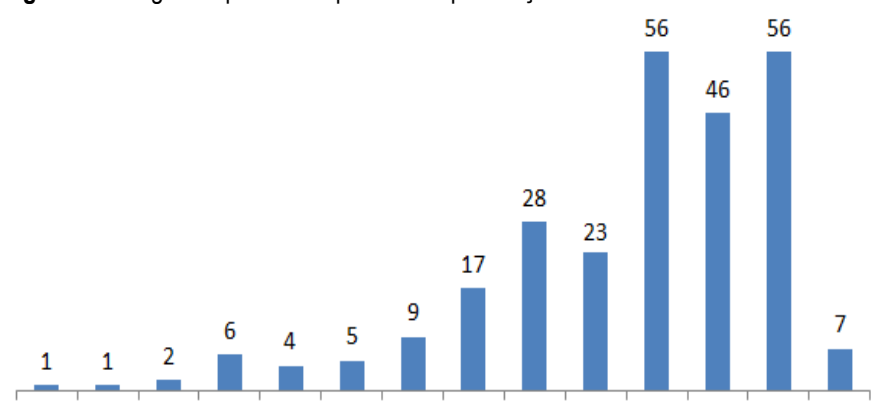

20012002200520072008200920102011201220132014201520162017

Fonte: As autoras.

\section{Resultados}

\section{Métricas preliminares}

Para análise das pesquisas aderentes, estas foram primeiramente classificadas pelos tópicos gerais, que consistem em tipos de envoltória verde ao qual cada pesquisa se refere (Figura 3). Analisando este resultado, observa-se que o tipo de envoltória predominante varia conforme a base de dados e que as pesquisas raramente tratam de duas ou todas as tipologias de envoltórias verdes.

Figura 3 - Tópicos gerais por base de dados

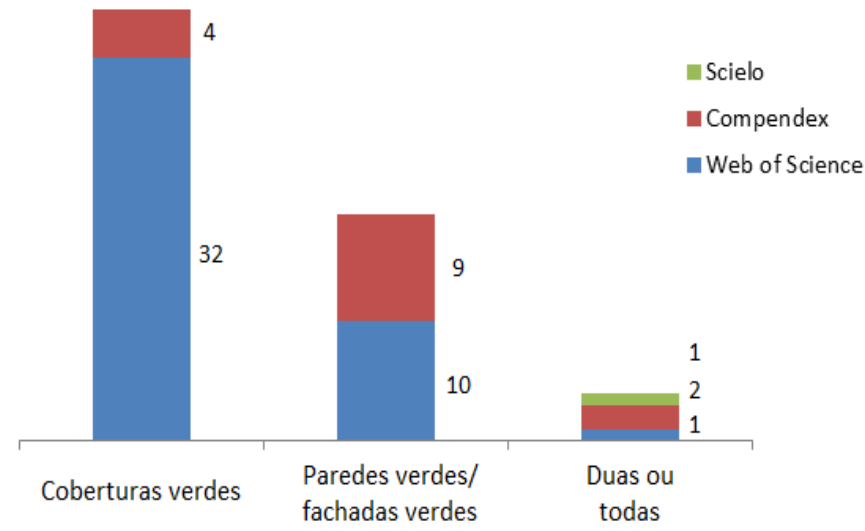

Fonte: As autoras.

Em um segundo momento, aprimorando a classificação anterior para tipos de pesquisa, observa-se que as pesquisas são em sua maioria experimentos, seguidos por simulações e cálculos/modelos de avaliação (Figura 4). Entre as pesquisas que definiram cálculos/modelos de avaliação, nenhuma englobou coberturas e paredes ou fachadas verdes em conjunto, o que pode ser justificado pelas três tipologias serem distintas em diversas características de implantação e funcionamento (MALYS; MUSY; INARD, 2016; MATHEUS et al., 2015).

As pesquisas aderentes provêm, em sua maior parte, das regiões da Ásia, Europa/Oriente Médio (Mediterrâneo), 
América do Norte e Oceania, basicamente nesta ordem em relação à quantidade de artigos encontrados. Apenas um destes artigos provém do Brasil; vale ressaltar, contudo, o fato de que a base de dados utilizada para encontrar pesquisas brasileiras em português (Scielo) possui restrição de alcance, não encontrando artigos de todos os periódicos científicos brasileiros. Da mesma forma, as outras bases de dados também possuem restrições, não encontrando todos os artigos existentes.

Figura 4 - Classificação por tipos de pesquisa e tópicos gerais

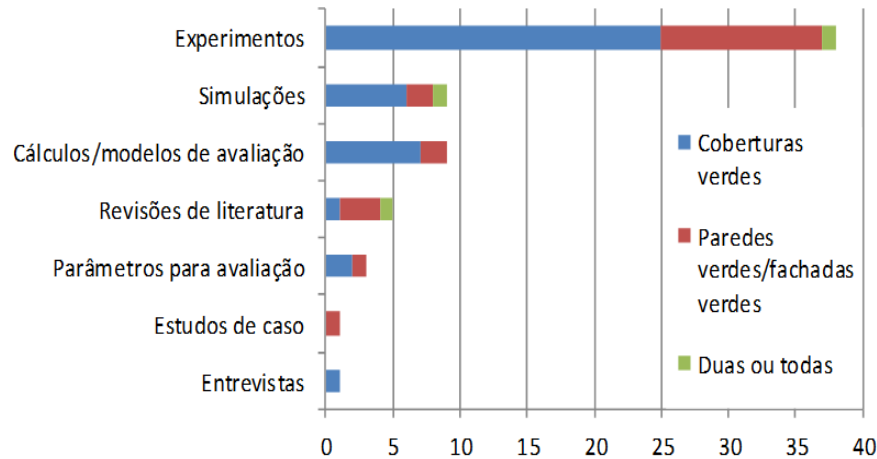

Fonte: As autoras.

\section{Análise dos textos - Temperatura interna das edificações}

Através da leitura das pesquisas aderentes, foi possível classificar os resultados de modo a resolver a questão determinada pela presente pesquisa, qual seja, a influência que as envoltórias vegetadas desempenham na temperatura interna das edificações, bem como quais são as variáveis de desempenho.

As pesquisas mensuraram as variações de temperatura de diversas formas, tanto com medições da temperatura de superfície interna da envoltória (faces internas de paredes ou lajes sob a influência de envoltória verde), quanto medindo a temperatura do ar do ambiente interno a diferentes alturas, incluindo a altura do usuário. A presente pesquisa focou em apresentar quantitativamente a influência das envoltórias verdes na temperatura interna do ar das edificações, por ser a informação mais relevante sob o ponto de vista da sensação térmica do usuário.

As pesquisas que quantificaram as reduções da temperatura interna do ar foram classificadas no Quadro 1. Houve diferença significativa entre a redução de temperatura proporcionada pelas coberturas verdes e paredes verdes.

As coberturas verdes dos quatro resultados indicados no Quadro 1 são coberturas extensivas, com até $20 \mathrm{~cm}$ de espessura, com exceção de Yang et al. (2015), que estudaram duas variações: uma delas possuía $10 \mathrm{~cm}$ a mais de camada de terra. O estudo concluiu que esta diferença não resultou em melhoria significativa no isolamento térmico. Tam, Wang e Le (2016) estudaram três coberturas verdes, sendo uma delas intensiva, porém o valor indicado no Quadro 1 é a redução máxima da temperatura do ar atingida pela cobertura extensiva de melhor desempenho deste estudo. A escolha deste valor para a tabela teve o objetivo de fazer a comparação com os dados das demais coberturas de características semelhantes, presentes no Quadro 1. A cobertura verde intensiva promoveu resfriamento de 2,7 a $3,4^{\circ} \mathrm{C}$ (TAM; WANG; LE, 2016).

Nas pesquisas citadas no Quadro 1, a redução de temperatura das coberturas verdes foi calculada comparando as coberturas verdes com coberturas de laje impermeabilizada de concreto, tratadas com camada de material de isolamento térmico. A exceção foi 0 experimento de Kumar e Mahalle (2016), cuja laje impermeabilizada de concreto não possuía qualquer material adicional de isolamento térmico. Isto pode ser uma explicação à maior redução de temperatura obtida por Kumar e Mahalle em relação às outras pesquisas de coberturas verdes.

Entre as pesquisas citadas no Quadro 1 sobre paredes verdes, as paredes utilizadas não possuíam isolamento térmico adicional, com exceção do experimento de Olivieri, Olivieri e Neila (2014) ${ }^{1}$. No experimento de Haggag, Hassan e Elmasry (2014), a parede consistia em blocos de concreto oco de espessura $20 \mathrm{~cm}$, com $2 \mathrm{~cm}$ de espessura de estuque branco de cada lado da parede. $\mathrm{Na}$ simulação de Malys, Musy e Inard (2016), a parede consistia em uma única camada de concreto, com $10 \mathrm{~cm}$ de espessura.

As paredes verdes do Quadro 1 referem-se apenas ao modelo de parede verde de vasos de planta perimetrais distribuídos ao longo da parede, descrito por Dunnett e Kingsbury (2004) e Ottelé (2011). As fachadas verdes, conforme descritas por Dunnett e Kingsbury (2004) e Ottelé (2011) não estão incluídas, apesar de Haggag, Hassan e Elmasry (2014) utilizarem a nomenclatura green façades no título da pesquisa.

Quadro 1 - Redução de temperatura interna do ar de ambientes com envoltórias verdes

\begin{tabular}{|c|c|l|}
\hline Envoltória & $\begin{array}{c}\text { Redução de } \\
\text { temperatura }\end{array}$ & \multicolumn{1}{c|}{ Referência } \\
\hline \multirow{2}{*}{$\begin{array}{c}\text { Coberturas } \\
\text { verdes } \\
\text { extensivas }\end{array}$} & $\leq 2,1^{\circ} \mathrm{C}$ & (TAM; WANG; LE, 2016) \\
\cline { 2 - 3 } & $\leq 4,4^{\circ} \mathrm{C}$ & (KUMAR; MAHALLE, 2016) \\
\cline { 2 - 3 } & 0,5 a $0,95^{\circ} \mathrm{C}$ & (YANG et al., 2015) \\
\hline \multirow{3}{*}{$\begin{array}{c}\text { Paredes } \\
\text { verdes }\end{array}$} & $5^{\circ} \mathrm{C}$ (média) & $\begin{array}{l}\text { (HAGGAG; HASSAN; ELMASRY, } \\
\text { 2014) }\end{array}$ \\
\cline { 2 - 3 } & $4,1^{\circ} \mathrm{C}$ (média) & (OLIVIERI; OLIVIERI; NEILA, 2014) \\
\cline { 2 - 3 } & $3 \mathrm{a} 7,5^{\circ} \mathrm{C}$ & (MALYS; MUSY; INARD, 2016) \\
\hline
\end{tabular}


As reduções apresentadas no Quadro 1 referem-se às máximas reduções diurnas, que ocorrem durante o período da tarde; os horários variam conforme a localidade estudada em cada pesquisa. O motivo é a redução do pico de temperatura da superfície externa da laje ou parede, relacionada principalmente à insolação. Os componentes da envoltória verde absorvem o calor promovido pela insolação, provocando atraso térmico na laje ou parede.

Além das reduções diurnas de temperatura, outro efeito relevante das envoltórias verdes na temperatura interna das edificações é a inércia térmica. A amplitude térmica dos ambientes com a envoltória verde é menor (KUMAR; MAHALLE, 2016). Isto acontece devido ao calor absorvido pela envoltória ser dissipado de forma mais lenta do que seria em uma laje ou parede exposta, o que também pode acarretar em aquecimento do ambiente interno (YANG et al., 2015). O que determinará se um aquecimento indesejado ocorrerá durante a noite é a variável climática, somada à espessura dos materiais envolvidos e suas respectivas condutividades térmicas. $\mathrm{O}$ comportamento térmico de edificações sem a influência de condicionamento ativo depende muito da inércia térmica dos materiais da envoltória (GAGLIANO et al., 2015).

No caso de uso de coberturas verdes para isolamento térmico em climas frios, dependendo do clima, o efeito noturno da inércia térmica pode não ser suficiente, sendo necessário o uso de isolamento térmico adicional. Segundo Squier e Davidson (2016) em experimento para o clima de Syracuse, Estados Unidos, a cobertura verde não pode substituir o isolamento térmico.

Todas as pesquisas citadas no Quadro 1 observaram efeitos de inércia térmica superiores nas envoltórias verdes em relação às envoltórias não-vegetadas, com exceção da pesquisa de Olivieri, Olivieri e Neila (2014). Este experimento não apresentou inércia térmica superior ao caso sem vegetação no período da noite; isto se deve ao fato de que a constituição de sua envoltória vegetada envolve materiais de resistência térmica inferior, conforme visto anteriormente. Neste caso, a perda noturna de calor parece ter sido causada pela maior umidade do substrato da parede verde, que foi o que propiciou a maior diferença de resistência térmica observada pelo estudo entre o caso vegetado e o nãovegetado.

Os fenômenos que compõem o balanço energético de uma cobertura verde são as trocas térmicas por radiação e convecção, evapotranspiração do solo e das plantas e a evaporação/condensação do vapor de água, bem como a condução térmica e armazenamento de calor na camada de solo (GAGLIANO et al., 2015). Nas envoltórias verdes verticais (paredes verdes e fachadas verdes), os fenômenos citados acima também ocorrem, mesmo que com diferentes proporções em relação às coberturas verdes. Wong et al. (2010) afirmam que variações no desempenho térmico das envoltórias verdes verticais ocorrem conforme a tipologia utilizada, sendo as variações de paredes verdes do seu estudo mais eficientes do que a fachada verde também presente em seu estudo ${ }^{2}$.

Gagliano et al. (2015) afirmam, com base em resultados experimentais, que o desempenho das coberturas verdes foi superior ao da cobertura de alta refletância na questão da temperatura interna do ar (até $2,5^{\circ} \mathrm{C}$ de redução), e a maior redução de temperatura $\left(3,5^{\circ} \mathrm{C}\right)$ foi em relação à cobertura de concreto com apenas uma camada de isolamento térmico. A adição de uma segunda camada de isolamento térmico de $4 \mathrm{~mm}$ (material não especificado, apenas espessura e suas propriedades térmicas) não auxiliou no desempenho $\mathrm{e}$ inclusive reduziu o desempenho total da cobertura analisada, por não permitir o resfriamento noturno necessário para o clima aonde foi realizada a pesquisa (GAGLIANO et al., 2015). Isto demonstra a relevância da correta especificação do isolamento térmico, sendo mais uma variável a ser observada.

\section{Análise dos textos - Variáveis de desempenho térmico}

As variáveis de desempenho térmico encontradas estão descritas no Quadro 2. As variáveis estudadas em maior número de artigos não são, necessariamente, aquelas de maior relevância. As características climáticas nem sempre são citadas propriamente como uma variação, visto que os experimentos ou simulações são realizados com envoltórias verdes cujas características são adequadas ao clima do local do experimento ou simulação em questão. Portanto, a questão climática pode ser a variável de maior notoriedade entre todas, como destacam Lin et al. (2013) ao afirmarem que a eficiência térmica de cobertura verde varia em diferentes climas, tendo Susorova et al. (2013) dito o mesmo a respeito das paredes verdes. Peng e Jim (2015) afirmam que os parâmetros do clima são a principal causa de variações sazonais e diurnas no desempenho térmico das coberturas verdes.

De acordo com Peng e Jim (2015), 83.6\% a 86\% das variações de efeito térmico das coberturas verdes estão relacionadas com a temperatura do ar, umidade relativa, velocidade do vento, radiação solar e umidade do solo; entre as cinco características descritas por Peng e Jim, quatro são características climáticas. 
Quadro 2 - Variáveis de desempenho térmico das coberturas e paredes verdes

\begin{tabular}{|c|c|c|}
\hline $\begin{array}{l}\text { Variáveis de } \\
\text { desempenho }\end{array}$ & Principais observações & $\begin{array}{l}\text { No. De } \\
\text { artigos }\end{array}$ \\
\hline $\begin{array}{l}\text { Características da } \\
\text { vegetação }\end{array}$ & $\begin{array}{l}\text { A maior densidade foliar influencia positivamente (CAMERON; TAYLOR; EMMETT, 2014; CHAN; CHOW, } \\
\text { 2013; CHEN; LI; LIU, 2013; CHEN; TSAY; CHIU, 2017; MONTEIRO et al., 2016; SUSOROVA et al., 2013); } \\
\text { A maior densidade foliar melhora o isolamento térmico da cobertura verde através do sombreamento (TAM; } \\
\text { WANG; LE, 2016); } \\
\text { A maior taxa de evapotranspiração influencia positivamente (CAMERON; TAYLOR; EMMETT, 2014; PODDAR; } \\
\text { PARK; CHANG, 2016; ZHAO et al., 2014) }\end{array}$ & 13 \\
\hline Umidade do substrato & $\begin{array}{l}\text { Em cobertura verde com substrato seco, não há resfriamento por evapotranspiração (BEVILACQUA et al., } \\
\text { 2015); } \\
\text { Substrato úmido aumentou a condutividade térmica (LIN et al., 2013; PIANELLA et al., 2016); } \\
\text { O desempenho térmico das coberturas verdes para resfriamento, através da evaporação do substrato e da } \\
\text { evapotranspiração das plantas, depende fortemente da quantidade de água disponível no substrato } \\
\text { (GAGLIANO et al., 2015) }\end{array}$ & 9 \\
\hline $\begin{array}{l}\text { Características } \\
\text { climáticas }\end{array}$ & $\begin{array}{l}\text { Há variação conforme a zona climática no desempenho de coberturas verdes (LIN et al., 2013) e fachadas } \\
\text { verdes (SUSOROVA et al., 2013); } \\
\text { Cobertura verde apresentou efeito de resfriamento no verão, outono e primavera, e aquecimento no inverno } \\
\text { (PENG; JIM, 2015) } \\
\text { Em locais onde a velocidade do vento é baixa, a vegetação (paredes verdes em frente a esquadrias abertas) é } \\
\text { altamente recomendada para resfriamento de ambientes internos (CHEN; TSAY; CHIU, 2017) }\end{array}$ & 8 \\
\hline $\begin{array}{l}\text { Composição do } \\
\text { substrato }\end{array}$ & $\begin{array}{l}\text { Substratos porosos aumentam a capacidade térmica (JIM, 2014); } \\
\text { Falta de sombreamento na cobertura verde faz com que o conforto térmico dependa muito do material do } \\
\text { substrato (PÉREZ et al., 2015) }\end{array}$ & 7 \\
\hline Sobreamento & $\begin{array}{l}\text { Fachada verde: variações notáveis por conta da insolação, promovendo maior resfriamento em paredes com } \\
\text { maior insolação do que em paredes mais sombreadas que a recebam (JIM, 2015); } \\
\text { Cobertura verde é mais úmida com menos insolação (BUCKLAND-NICKS; HEIM; LUNDHOLM, 2016) }\end{array}$ & 5 \\
\hline Espessura do substrato & $\begin{array}{l}\text { Substratos mais profundos aumentam a capacidade térmica (CHAN; CHOW, 2013; JIM, 2014; TAM; WANG; } \\
\text { LE, 2016); } \\
\text { Substratos mais finos ( } 5 \mathrm{~cm} \text { ) são mais suscetíveis à evaporação e consequentemente ficam mais secos e } \\
\text { quentes (REYES et al., 2016) }\end{array}$ & 5 \\
\hline Transpiração & $\begin{array}{l}\text { Em fachada verde, transpiração influenciou em proporções menores do que as do sombreamento } \\
\text { (HOELSCHER et al., 2016); } \\
\text { Em fachada verde, transpiração resfriou o ar mais do que sombreamento e isolamento térmico (JIM, 2015) } \\
\text { Em cobertura verde, a evapotranspiração promoveu resfriamento evaporativo que, por sua vez, aumentou a } \\
\text { resistência térmica da cobertura (OULDBOUKHITINE; SPOLEK; BELARBI, 2014) }\end{array}$ & 4 \\
\hline Camada de ar & $\begin{array}{l}\text { Parede verde com camada selada de ar entre esta e a parede possui desempenho melhor no resfriamento da } \\
\text { parede do que se a camada de ar possuir ventilação natural (CHEN; LI; LIU, 2013); }\end{array}$ & 1 \\
\hline Isoalmento & $\begin{array}{l}\text { A adição de uma segunda camada de isolamento térmico de } 4 \text { mm não auxiliou no desempenho da cobertura } \\
\text { verde e inclusive reduziu o desempenho total da cobertura analisada (GAGLIANO et al., 2015) }\end{array}$ & 1 \\
\hline
\end{tabular}

Fonte: As autoras.

A afirmação de Peng e Jim (2015) proporciona um entendimento maior acerca das variáveis. A única característica que não envolve variáveis climáticas entre as citadas como principais envolve a umidade do substrato, que é, entretanto, influenciada diretamentepelas variáveis climáticas em si e pelas características da vegetação. A espessura, composição do substrato e insolação também influenciam na umidade deste, assim como a transpiração também está relacionada à umidade (Quadro 2). Portanto, todas as variáveis citadas no Quadro 2 são significativas e estão relacionadas de alguma forma com a umidade do substrato. $\mathrm{O}$ isolamento térmico da laje é uma possível exceção, por não terem sido encontradas mais pesquisas que proporcionem um entendimento mais amplo sobre as dinâmicas envolvidas nesta variável especificamente relacionada às coberturas verdes.

As afirmações de pesquisas acerca da umidade do substrato são destacáveis por revelarem uma lacuna. Em substrato seco, o resfriamento evaporativo não acontece (Quadro 2). Por outro lado, em substrato úmido, apesar de haver resfriamento evaporativo, a condutividade térmica aumenta (Quadro 2). Desta forma, foi encontrada uma lacuna a respeito do peso de ambas as características no resfriamento. Este peso poderia determinar a umidade ideal do ponto de vista do desempenho térmico e também auxiliar no entendimento da variação de desempenho relacionada à insolação da cobertura verde, que também influencia na umidade do substrato (BUCKLANDNICKS; HEIM; LUNDHOLM, 2016), entre outras variações relacionadas. Como a evaporação do substrato e a evapotranspiração das plantas dependem fortemente da quantidade de água disponível no substrato e são fenômenos que promovem resfriamento (GAGLIANO et al., 2015), é evidente a importância desta característica no desempenho global de resfriamento das envoltórias verdes. 
A composição do substrato se torna uma variável de maior importância quando o substrato está mais exposto. Pérez et al. (2015) observaram uma cobertura verde de pouco sombreamento, com apenas $10 \%$ de cobertura por vegetação; esta falta de sombreamento faz com que o conforto térmico dependa muito do material do substrato (Quadro 2). Desta forma, as variáveis "composição do substrato" e "sombreamento" também influenciam uma sobre a outra.

Sobre a transpiração, seu peso em fachadas verdes de características similares pode ser oposto (Quadro 2). Em um estudo em Hong Kong (JIM, 2015), este fenômeno possui maior influência no resfriamento do ar do que sombreamento e isolamento térmico em fachadas verdes, enquanto que em outro estudo, na Alemanha (HOELSCHER et al., 2016), é destacado o sombreamento com maior influência do que a transpiração. As condições climáticas distintas dos dois locais estudados podem explicar a diferença entre os resultados, assim como a insolação recebida pelas duas fachadas verdes. A utilização de diferentes espécies de vegetação também pode influenciar na transpiração das mesmas (CAMERON; TAYLOR; EMMETT, 2014). Assim sendo, e considerando a análise das demais variáveis de desempenho, fica evidente a relação intrínseca e multifacetada entre estas. Isto impossibilita a determinação de hierarquia entre as variáveis, visto que a maioria destas afeta diretamente o desempenho de outras, podendo uma única variável influenciar profundamente $o$ desempenho global e prejudicar seu funcionamento.

\section{Análise dos textos - Economia de energia}

Entre as pesquisas aderentes, $30 \%$ mensuram a economia de energia gerada pelas envoltórias verdes em relação ao condicionamento ativo (Quadro 3), para resfriamento ou aquecimento, predominando o resfriamento. As pesquisas que fizeram observações negativas em relação à economia de energia tratam da questão comentando sobre as características que foram classificadas no Quadro 2 como variáveis de desempenho, cuja complexidade foi analisada na subseção anterior.

Algumas das pesquisas com resultados positivos também citaram variáveis que podem diminuir a economia de energia, como Pérez et al. (2017), que afirmam que as paredes com maior insolação devem receber as envoltórias verdes, para que a economia de energia para resfriamento seja eficiente.

A economia de energia das coberturas verdes apontada pelos estudos do Quadro 3 é em relação a coberturas convencionais de laje impermeabilizada (FOUSTALIERAKI et al., 2017; YANG et al., 2015). Entre os artigos estudados sobre economia de energia e demais artigos sobre o comportamento térmico das coberturas verdes, não foram encontrados estudos comparativos de coberturas verdes e coberturas mais utilizadas do Brasil, que são as de telhas cerâmicas ou de fibrocimento com camada de ar entre estas e a laje, o que caracteriza uma lacuna no conhecimento atual. Convém, entretanto, explicitar que houve restrições nas buscas de artigos, relacionadas tanto à limitação da pesquisa aos últimos cinco anos e filtros para seleção de artigos, quanto ao alcance das bases de dados, principalmente da base Scielo. Um exemplo de artigo que não consta na busca é o de Parizotto e Lamberts (2011), que se trata de um experimento que comprovou a contribuição das coberturas verdes com benefícios térmicos e eficiência energética em clima temperado, em comparação com telhado cerâmico e metálico.

\begin{tabular}{|c|c|c|c|}
\hline $\begin{array}{l}\text { Economia } \\
\text { de energia }\end{array}$ & \multicolumn{2}{|c|}{ Principais observações } & $\begin{array}{l}\text { No. De } \\
\text { artigos }\end{array}$ \\
\hline \multirow[b]{2}{*}{$\begin{array}{c}\text { Observações } \\
\text { positivas }\end{array}$} & $\begin{array}{l}\text { Paredes } \\
\text { verdes/ } \\
\text { fachadas } \\
\text { verdes }\end{array}$ & $\begin{array}{l}\text { Até } 37 \% \text { de economia anual de } \\
\text { energia com parede verde } \\
\text { (DJEDJIG et al., 2017); } \\
\text { Até } 34 \% \text { de economia de } \\
\text { energia com fachada verde } \\
\text { (PÉREZ et al., 2017); } \\
\text { Economia diária de 16\% com } \\
\text { paredes verdes nos dois } \\
\text { meses mais quentes do ano } \\
\text { (PAN; CHU, 2016); }\end{array}$ & \multirow[b]{2}{*}{17} \\
\hline & $\begin{array}{l}\text { Coberturas } \\
\text { verdes }\end{array}$ & $\begin{array}{c}\text { Até } 15,2 \% \text { de economia anual } \\
\text { de energia com cobertura verde } \\
\text { (YANG et al., 2015); } \\
\text { 15,1\% de economia anual de } \\
\text { energia com cobertura verde } \\
\text { (FOUSTALIERAKI et al., 2017) } \\
\text { Coberturas verdes e cool roofs } \\
\text { promovem maior economia e } \\
\text { benefícios ambientais do que } \\
\text { coberturas com grande } \\
\text { isolamento térmico } \\
\text { (GAGLIANO et al., 2015) }\end{array}$ & \\
\hline $\begin{array}{l}\text { Observações } \\
\text { negativas }\end{array}$ & \multicolumn{2}{|c|}{$\begin{array}{l}\text { O balanço energético das coberturas verdes é } \\
\text { complexo e nem sempre resulta em economia } \\
\text { de energia (MOODY: SAlLOR. 2013) }\end{array}$} & 3 \\
\hline
\end{tabular}

Fonte: As autoras.

Quanto às paredes-controle dos estudos de paredes verdes, o experimento de Djedjig et al. (2017) era constituído de parede de $20 \mathrm{~cm}$ de espessura feita de blocos de concreto; o de Pérez et al. (2017) era uma parede de blocos cerâmicos alveolares de $30 \times 19 \times 29$ $\mathrm{cm}$, com acabamento de gesso internamente e de argamassa de cimento externamente. No de Pan e Chu (2016), não há informação sobre o material da parede, apenas o dado de que na parede em questão havia uma esquadria que foi selada com duas camadas de placas de gesso de $19 \mathrm{~mm}$, com forro de lã de vidro entre elas; é informado também que estes materiais que selaram a esquadria possuem a mesma condutividade térmica da parede da edificação (PAN; CHU, 2016). 
Sob o ponto de vista da economia de energia com condicionamento ativo, as coberturas verdes são preferíveis a coberturas reflexivas no local estudado por Lee, Ryu e Jiang (2015) ${ }^{3}$. A variável responsável por este resultado, destacada pelo estudo, foi a irrigação adequada da cobertura verde, enquanto a quantidade de nuvens foi uma variável que reduziu a eficiência da cobertura reflexiva (LEE; RYU; JIANG, 2015). Perini e Magliocco (2014) afirmam que coberturas verdes são mais eficientes na economia de energia da edificação do que vegetação no solo (grama, arbustos e árvores), e destacam a densidade de folhagem como principal variável neste desempenho.

Os resultados coletados sobre economia de energia do Quadro 3 são comparáveis àqueles da variação de temperatura do ar interno na relação cobertura verde/parede verde do Quadro 1. É possível perceber um desempenho superior das paredes verdes em relação às coberturas verdes, apesar destes dados serem de pesquisas diferentes e estas análises comparativas serem apenas considerações. A exceção é o resultado de Pan e Chu (2016) do Quadro 3, referente a paredes verdes, cuja porcentagem de economia de energia possui valor mais próximo ao valor de economia encontrado para as coberturas verdes, também do Quadro 3.

Analisando os métodos de Pan e Chu (2016), a orientação da parede verde estudada foi sudoeste, que em Hong Kong (local do experimento) é a de maior radiação solar. Portanto, a orientação da parede não aparenta ser o motivo da discrepância nos resultados. Analisando a planta baixa da edificação do experimento, observa-se que o ambiente analisado é de área reduzida, com 6,65 $\mathrm{m}^{2}$, com outras duas faces inteiras voltadas ao exterior, sendo que uma delas, voltada a sudeste, possui esquadria envidraçada. A face da parede verde também possui esquadria (PAN; CHU, 2016). Mesmo que duas camadas de placas de gesso de $19 \mathrm{~mm}$ tenham sido aplicadas nas áreas das esquadrias de modo a isolá-las e uma camada de $19 \mathrm{~mm}$ de placas de espuma de poliestireno tenha sido aplicada em ambas as faces internas das paredes expostas (com exceção daquela aonde foi aplicada a parede verde), o isolamento pode não ter sido suficiente, o que pode ter influenciado negativamente o resultado.

Entretanto, além do experimento, Pan e Chu (2016) realizaram simulações computacionais do mesmo ambiente sob influência de duas variáveis; uma parede verde similar à do experimento e uma cobertura verde. $\mathrm{Na}$ simulação, a economia de energia com condicionamento ativo da parede verde foi similar à do experimento realizado; porém, a economia da cobertura verde simulada foi de apenas metade da economia da parede verde existente (PAN; CHU, 2016).
Desta forma, a economia de energia encontrada por Pan e Chu (2016) é reduzida em relação às demais por influência de características atípicas do ambiente analisado em relação às outras pesquisas, mas apresenta a proporção de economia entre cobertura verde e parede verde similar à média dos resultados das demais pesquisas.

\section{Demais observações}

Através da revisão sistemática de literatura, foi possível compreender o comportamento térmico de ambientes internos sob a influência das envoltórias verdes. Este foi traçado através de levantamento de valores de redução das temperaturas diurnas e demais características relacionadas à inércia térmica, assim como foram identificadas diversas variáveis de desempenho e suas dinâmicas.

O mapeamento sistemático apresentado, prévio à revisão sistemática, promoveu o entendimento do contexto em que se inserem as pesquisas, tornando evidente a atualidade do tema e o seu número crescente nos últimos anos. O mapeamento também permitiu justificar a escolha do período analisado, por ser o de maior número de pesquisas relacionadas ao tema.

Os artigos com experimentos ou simulações de resultados similares são úteis para a análise sistemática, tornando possível a comparação entre os métodos utilizados para identificar as variáveis que podem ter influenciado as diferenças nos resultados. Isto também torna mais embasadas as afirmações realizadas em uma revisão sistemática.

A quantificação da redução de temperatura observada nesta revisão, bem como a quantificação da economia de energia, não podem ser utilizadas como parâmetros para grande parte das construções brasileiras. Estudos sobre coberturas verdes em que o caso-controle seja cobertura cerâmica (em sua diversidade de formatos de telha, cores, etc.) ou de fibrocimento sobre laje são necessários no Brasil ou em locais de climas semelhantes aos brasileiros.

Outra lacuna relevante é sobre a questão da umidade do substrato, do seu peso no resfriamento. A resposta a esta lacuna será complexa, devido à influência que a umidade do substrato exerce sobre outras características da envoltória verde também responsáveis pelo resfriamento (GAGLIANO et al., 2015) e estas, por sua vez, sofrem grande influência das condições climáticas locais (LIN et al., 2013). De acordo com a análise desta revisão, quase todas as variáveis de desempenho estão relacionadas com a umidade do substrato, o que destaca ainda mais a relevância desta variável.

Uma variável bastante presente nos artigos aderentes desta pesquisa é em relação às diversas características 
climáticas, visto que os artigos provêm de diferentes locais do globo. Isto proporcionou compreensão das dinâmicas de diferentes características climáticas e sua influência nas dinâmicas do sistema de envoltória verde.

As variáveis de desempenho, tanto climáticas quanto das características do sistema de envoltória, são diversas e intrinsecamente relacionadas, o que impossibilita o entendimento de fenômenos isolados sem que se tenha conhecimento de todas estas variáveis. Por isso, os motivos pelos quais as paredes verdes apresentaram redução de temperatura do ar superior às coberturas verdes (Quadro 1) são de difícil identificação e podem ser diversos.

De início, ao analisar as variáveis de desempenho, as variáveis climáticas parecem ter maior influência no desempenho das envoltórias verdes, sendo, inclusive, o que alguns autores afirmam (PENG; JIM, 2015). Analisando cada variável, climática ou não, e as relações multifacetadas que exercem entre si, ficou evidente que a determinação de hierarquia entre estas não é possível e nem prudente. A análise da coerência entre as variáveis é mais importante do que a determinação de sua hierarquia, bem como esta ação deve preceder a definição da tipologia e características de uma determinada envoltória a ser implantada em dado lugar.

Os resultados apresentados neste artigo referem-se ao universo de pesquisa traçado, incluindo as possíveis lacunas identificadas. $\mathrm{O}$ enfoque da pesquisa apenas nos últimos cinco anos, os filtros para seleção de artigos e a base de dados brasileira escolhida podem constituir uma restrição ao alcance da pesquisa.

\section{Conclusões}

A revisão sistemática de literatura permitiu responder à questão estabelecida, que consistia em determinar a influência que as envoltórias vegetadas desempenham na temperatura interna das edificações e identificar as variáveis de desempenho térmico. Além disso, foram verificados pontos frágeis e lacunas do conhecimento atual.

Os trabalhos analisados mostram que a redução de temperatura do ambiente interno é significativa, sendo mais acentuada no caso de paredes verdes. O desempenho térmico das envoltórias verdes depende de variáveis como o tipo de vegetação, densidade de folhagem, composição do substrato, etc., destacando as características climáticas, apesar da maioria das pesquisas focar em resultados de outras variáveis. As lacunas do universo selecionado para a presente revisão de literatura envolvem $o$ peso da umidade do substrato no resfriamento de coberturas verdes e a comparação do desempenho de coberturas verdes com coberturas de telhas cerâmicas ou de fibrocimento.

A análise das variáveis identificou que estas exercem entre si relações multifacetadas, o que tornou evidente que a análise da coerência entre as variáveis é mais importante do que a determinação de sua hierarquia. A definição da tipologia e características de uma determinada envoltória a ser implantada em dado lugar deve partir desta ação.

Em estudos futuros, o cruzamento das informações obtidas neste artigo com resultados a respeito do desempenho térmico externo à edificação pode esclarecer pontos importantes, como verificar se as características ideais para o desempenho interno coincidem com as características ideais para a otimização do desempenho externo, bem como avaliar se as variáveis de desempenho são as mesmas. Estas informações podem complementar e tornar mais amplo o entendimento das dinâmicas que envolvem o desempenho térmico das envoltórias verdes.

\section{Agradecimentos}

As autoras agradecem à Fundação CAPES pelo apoio financeiro à pesquisa através de bolsa de mestrado pelo Programa de PósGraduação em Arquitetura, Tecnologia e Cidade da Universidade Estadual de Campinas.

\section{Notas}

(1) O experimento citado possuía como parede duas camadas de aço galvanizado $(0,001 \mathrm{~m}$ cada) e feltro de poliéster $(0,0015$ $\mathrm{m}$ cada), com uma camada de poliestireno extrudado $(0,07 \mathrm{~m})$, somando-se a estas o substrato presente tanto na envoltória vegetada quanto na não-vegetada. A resistência térmica é de $0,13 \mathrm{~m}^{2} \mathrm{~K} / \mathrm{W}$, tanto interna quanto externa em ambas envoltórias, com exceção da envoltória não vegetada que possui resistência térmica externa de $0,4 \mathrm{~m}^{2} \mathrm{~K} / \mathrm{W}$, cuja diferença se deve ao substrato seco apresentado com resistência térmica significativamente superior ao substrato com vegetação (OLIVIERI; OLIVIERI; NEILA, 2014). A vegetação não entrou como variável entre as camadas descritas.

(2) Vale ressaltar que a fachada verde do experimento de Wong et al. (2010) possuía folhagem um tanto escassa, o que pode ter influenciado nos resultados. 
(3) Apesar de Lee, Ryu e Jiang (2015) concluírem que a cobertura reflexiva é preferível sob o ponto de vista da mitigação do clima e da facilidade de manutenção.

\section{Referências}

ABDUL-RAHMAN et al. Vertical greenery systems (vgs) in urban tropics. Open House International, v. 39, n. 4, p. 42-52, dez. 2014.

AMBROSINI, D. et al. Evaluating mitigation effects of urban heat islands in a historical small center with the ENVI-Met ${ }^{\circledR}$ climate model. Sustainability, v. 6, n. 10, p. 7013-7029, 10 out. 2014. http://dx.doi.org/10.3390/su6107013

AZKORRA, Z. et al. Evaluation of green walls as a passive acoustic insulation system for buildings. Applied Acoustics, v. 89, p. 46-56, mar. 2015. https://doi.org/10.1016/j.apacoust.2014.09.010

BEVILACQUA, P. et al. Plant cover and floristic composition effect on thermal behaviour of extensive green roofs. Building and Environment, v. 92, p. 305-316, Outubro 2015. https://doi.org/10.1016/j.buildenv.2015.04.026

BRERETON, P. et al. Lessons from applying the systematic literature review process within the software engineering domain. Journal of Systems and Software, Software Performance5th International Workshop on Software and Performance. v. 80, n. 4, p. 571-583, Abril 2007. https://doi.org/10.1016/j.jss.2006.07.009

BUCKLAND-NICKS, M.; HEIM, A.; LUNDHOLM, J. Spatial environmental heterogeneity affects plant growth and thermal performance on a green roof. Science of The Total Environment, v. 553, p. 20-31, Maio 2016.

https://doi.org/10.1016/j.scitotenv.2016.02.063

CAMERON, R. W. F.; TAYLOR, J. E.; EMMETT, M. R. What's 'cool' in the world of green façades? How plant choice influences the cooling properties of green walls. Building and Environment, v. 73, p. 198-207, mar. 2014.

https://doi.org/10.1016/j.buildenv.2013.12.005

CHAN, A. L. S.; CHOW, T. T. Energy and economic performance of green roof system under future climatic conditions in Hong Kong. Energy and Buildings, v. 64, p. 182-198, Setembro 2013. https://doi.org/10.1016/j.enbuild.2013.05.015

CHEN, N.; TSAY, Y.; CHIU, W. Influence of vertical greening design of building opening on indoor cooling and ventilation. International Journal of Green Energy, v. 14, n. 1, p. 24-32, 2 jan. 2017. http://dx.doi.org/10.1080/15435075.2016.1233497

CHEN, Q.; LI, B.; LIU, X. An experimental evaluation of the living wall system in hot and humid climate. Energy and Buildings, v. 61, p. 298-307, jun. 2013. https://doi.org/10.1016/j.enbuild.2013.02.030

COMA, J. et al. Green roofs as passive system for energy savings in buildings during the cooling period: use of rubber crumbs as drainage layer. Energy Efficiency, v. 7, n. 5, p. 841-849, 8 abr. 2014. http://dx.doi.org/10.1007/s12053-014-9262-x

CONNELLY, M.; HODGSON, M. Experimental investigation of the sound absorption characteristics of vegetated roofs. Building and Environment, v. 92, p. 335-346, Outubro 2015. https://doi.org/10.1016/j.buildenv.2015.04.023

DJEDJIG, R. et al. Thermal effects of an innovative green wall on building energy performance. Mechanics \& Industry, v. 18, n. 1, p. 104, 2017. http://dx.doi.org/10.1051/meca/2016015

DUNNETT, N.; KINGSBURY, N. Planting green roofs and living walls. [s.l.] Timber Press, 2004.

ENGSTRÖM, E.; RUNESON, P. Software product line testing - A systematic mapping study. Information and Software Technology, v. 53, n. 1, p. 2-13, jan. 2011. http://dx.doi.org/10.1016/j.infsof.2010.05.011

FOUSTALIERAKI, M. et al. Energy performance of a medium scale green roof system installed on a commercial building using numerical and experimental data recorded during the cold period of the year. Energy and Buildings, v. 135, p. 33-38, 15 jan. 2017. http://dx.doi.org/10.1016/j.enbuild.2016.10.056

GAGLIANO, A. et al. A multi-criteria methodology for comparing the energy and environmental behavior of cool, green and traditional roofs. Building and Environment, v. 90, p. 71-81, Agosto 2015. http://dx.doi.org/10.1016/j.buildenv.2015.02.043 
HAGGAG, M.; HASSAN, A.; ELMASRY, S. Experimental study on reduced heat gain through green façades in a high heat load climate. Energy and Buildings, v. 82, p. 668-674, Outubro 2014. https://doi.org/10.1016/j.enbuild.2014.07.087

HARPER, G. E. et al. Nine-month evaluation of runoff quality and quantity from an experiential green roof in Missouri, USA. Ecological Engineering, The 13th Annual Conference of the American Ecological Engineering Society: Ecological Engineering and the Dawn of the 21st Century. v. 78, p. 127-133, Maio 2015. http://dx.doi.org/10.1016/j.ecoleng.2014.06.004

HOELSCHER, M.-T. et al. Quantifying cooling effects of facade greening: shading, transpiration and insulation. Energy and Buildings, SI: Countermeasures to Urban Heat Island. v. 114, p. 283-290, Fevereiro 2016. http://dx.doi.org/10.1016/j.enbuild.2015.06.047

JIM, C. Y. Passive warming of indoor space induced by tropical green roof in winter. Energy, v. 68, p. 272-282, Abril 2014. http://dx.doi.org/10.1016/j.energy.2014.02.105

JIM, C. Y. Thermal performance of climber greenwalls: effects of solar irradiance and orientation. Applied Energy, v. 154, p. 631643, Setembro 2015. https://doi.org/10.1016/j.apenergy.2015.05.077

KUMAR, V. V.; MAHALLE, A. M. Investigation of the thermal performance of green roof on a mild warm climate. International Journal of Renewable Energy Research, v. 6, n. 2, p. 487-493, 2016.

LA ROCHE, P.; BERARDI, U. Comfort and energy savings with active green roofs. Energy and Buildings, v. 82, p. 492-504, Outubro 2014. https://doi.org/10.1016/j.enbuild.2014.07.055

LEE, S.; RYU, Y.; JIANG, C. Urban heat mitigation by roof surface materials during the East Asian summer monsoon. Environmental Research Letters, v. 10, n. 12, p. 124012, 2015. http://dx.doi.org/10.1088/1748-9326/10/12/124012

LIN, B. B.; PHILPOTT, S. M.; JHA, S. The future of urban agriculture and biodiversity-ecosystem services: challenges and next steps. Basic and Applied Ecology, v. 16, n. 3, p. 189-201, Maio 2015. http://dx.doi.org/10.1016/j.baae.2015.01.005

LIN, B.-S. et al. Impact of climatic conditions on the thermal effectiveness of an extensive green roof. Building and Environment, v. 67, p. 26-33, Setembro 2013. http://dx.doi.org/10.1016/j.buildenv.2013.04.026

LUNDHOLM, J. T.; WEDDLE, B. M.; MACIVOR, J. S. Snow depth and vegetation type affect green roof thermal performance in winter. Energy and Buildings, v. 84, p. 299-307, Dezembro 2014. http://dx.doi.org/10.1016/j.enbuild.2014.07.093

LUO, H. et al. Carbon sequestration potential of green roofs using mixed-sewage-sludge substrate in Chengdu World Modern Garden City. Ecological Indicators, v. 49, p. 247-259, Fevereiro 2015. https://doi.org/10.1016/j.ecolind.2014.10.016

MADRE, F. et al. Green roofs as habitats for wild plant species in urban landscapes: first insights from a large-scale sampling. Landscape and Urban Planning, v. 122, p. 100-107, fev. 2014. https://doi.org/10.1016/j.landurbplan.2013.11.012

MALYS, L.; MUSY, M.; INARD, C. Direct and indirect impacts of vegetation on building comfort: a comparative study of lawns, green walls and green roofs. Energies, v. 9, n. 1, p. 32, jan. 2016. http://dx.doi.org/10.3390/en9010032

MARCHI, M. et al. Carbon dioxide sequestration model of a vertical greenery system. Ecological Modelling, Special Issue: Ecological Modelling for Ecosystem Sustainability: Selected papers presented at the 19th ISEM Conference, 28-31 October 2013, Toulouse, France. v. 306, p. 46-56, 24 jun. 2015. https://doi.org/10.1016/j.ecolmodel.2014.08.013

MATHEUS, C. et al. Desempenho térmico de envoltórias vegetadas em edificações no sudeste brasileiro. Ambiente Construído, v. 16, n. 1, p. 71-81, 8 set. 2015. http://dx.doi.org/10.1590/s1678-86212016000100061

MONTEIRO, M. V. et al. Relative importance of transpiration rate and leaf morphological traits for the regulation of leaf temperature. Australian Journal of Botany, v. 64, n. 1, p. 32-44, 15 mar. 2016. http://dx.doi.org/10.1071/BT15198

MOODY, S. S.; SAILOR, D. J. Development and application of a building energy performance metric for green roof systems. Energy and Buildings, v. 60, p. 262-269, Maio 2013. https://doi.org/10.1016/j.enbuild.2013.02.002

MULLER, J. N. et al. Diverse urban plantings managed with sufficient resource availability can increase plant productivity and arthropod diversity. Functional Plant Ecology, v. 5, p. 517, 2014. https://doi.org/10.3389/fpls.2014.00517 
OLIVIERI, F.; OLIVIERI, L.; NEILA, J. Experimental study of the thermal-energy performance of an insulated vegetal façade under summer conditions in a continental mediterranean climate. Building and Environment, v. 77, p. 61-76, jul. 2014. http://dx.doi.org/10.1016/j.buildenv.2014.03.019

OTTELÉ, M. The green building envelope: vertical greening. [s.1.] TU Delft, 2011.

OULDBOUKHITINE, S.-E.; SPOLEK, G.; BELARBI, R. Impact of plants transpiration, grey and clean water irrigation on the thermal resistance of green roofs. Ecological Engineering, v. 67, p. 60-66, jun. 2014. http://dx.doi.org/10.1016/j.ecoleng.2014.03.052

PAN, L.; CHU, L. M. Energy saving potential and life cycle environmental impacts of a vertical greenery system in Hong Kong: a case study. Building and Environment, v. 96, p. 293-300, 1 fev. 2016. https://doi.org/10.1016/j.buildenv.2015.06.033

PARIZOTTO, S.; LAMBERTS, R. Investigation of green roof thermal performance in temperate climate: A case study of an experimental building in Florianópolis city, Southern Brazil. Energy and Buildings, v. 43, n. 7, p. 1712-1722, 1 jul. 2011. https://doi.org/10.1016/j.enbuild.2011.03.014

PENG, L. L. H.; JIM, C. Y. Seasonal and diurnal thermal performance of a subtropical extensive green roof: the impacts of background weather parameters. Sustainability, v. 7, n. 8, p. 11098-11113, 14 ago. 2015. http://dx.doi.org/10.3390/su70811098

PÉREZ, G. et al. The thermal behaviour of extensive green roofs under low plant coverage conditions. Energy Efficiency, v. 8, n. 5, p. 881-894, 30 jan. 2015. http://dx.doi.org/10.1007/s12053-015-9329-3

PÉREZ, G. et al. Green facade for energy savings in buildings: the influence of leaf area index and facade orientation on the shadow effect. Applied Energy, v. 187, p. 424-437, Fevereiro 2017. http://dx.doi.org/10.1016/j.apenergy.2016.11.055

PERINI, K.; MAGLIOCCO, A. Effects of vegetation, urban density, building height, and atmospheric conditions on local temperatures and thermal comfort. Urban Forestry \& Urban Greening, v. 13, n. 3, p. 495-506, 2014.

http://dx.doi.org/10.1016/j.ufug.2014.03.003

PERINI, K.; ROSASCO, P. Is greening the building envelope economically sustainable? An analysis to evaluate the advantages of economy of scope of vertical greening systems and green roofs. Urban Forestry \& Urban Greening, v. 20, p. 328-337, Dezembro 2016. http://dx.doi.org/10.1016/j.ufug.2016.08.002

PIANELLA, A. et al. Steady-state and transient thermal measurements of green roof substrates. Energy and Buildings, v. 131, p. 123-131, 1 nov. 2016. http://dx.doi.org/10.1016/j.enbuild.2016.09.024

PODDAR, S.; PARK, D.; CHANG, S. Energy performance analysis of a dormitory building based on different orientations and seasonal variations of leaf area index. Energy Efficiency, p. 1-17, 3 nov. 2016. http://dx.doi.org/10.1007/s12053-016-9487-y

REYES, R. et al. Effect of substrate depth and roof layers on green roof temperature and water requirements in a semi-arid climate. Ecological Engineering, v. 97, p. 624-632, Dezembro 2016. http://dx.doi.org/10.1016/j.ecoleng.2016.10.025

SANTAMOURIS, M. Cooling the cities - A review of reflective and green roof mitigation technologies to fight heat island and improve comfort in urban environments. Solar Energy, v. 103, p. 682-703, Maio 2014.

https://doi.org/10.1016/j.solener.2012.07.003

SCHERER, M. J.; FEDRIZZI, B. M. Jardins verticais: potencialidades para o ambiente urbano. Revista Latino-Americana de Inovação e Engenharia de Produção, v. 2, n. 2, p. 49-61, 22 set. 2014. http://dx.doi.org/10.5380/relainep.v2i2.37883

SEBTI, A.; BENNIS, S.; FUAMBA, M. Optimization of the restructuring cost of an urban drainage network. Urban Water Journal, v. 13, n. 2, p. 119-132, 17 fev. 2016. https://doi.org/10.1080/1573062X.2014.923918

SHAFIQUE, M.; KIM, R. Low impact development practices: a review of current research and recommendations for future directions. Ecological Chemistry and Engineering S, v. 22, n. 4, 1 jan. 2015. https://doi.org/10.1515/eces-2015-0032

SQUIER, M.; DAVIDSON, C. I. Heat flux and seasonal thermal performance of an extensive green roof. Building and Environment, v. 107, p. 235-244, Outubro 2016. http://dx.doi.org/10.1016/j.buildenv.2016.07.025 
SUSOROVA, I. et al. A model of vegetated exterior facades for evaluation of wall thermal performance. Building and Environment, v. 67, p. 1-13, Setembro 2013. http://dx.doi.org/10.1016/j.buildenv.2013.04.027

TAM, V. W. Y.; WANG, J.; LE, K. N. Thermal insulation and cost effectiveness of green-roof systems: An empirical study in Hong Kong. Building and Environment, v. 110, p. 46-54, dez. 2016. https://doi.org/10.1016/j.buildenv.2016.09.032

VERSINI, P.-A. et al. Assessment of the hydrological impacts of green roof: from building scale to basin scale. Journal of Hydrology, v. 524, p. 562-575, Maio 2015. http://dx.doi.org/10.1016/j.jhydrol.2015.03.020

VIJAYARAGHAVAN, K.; JOSHI, U. M. Can green roof act as a sink for contaminants? A methodological study to evaluate runoff quality from green roofs. Environmental Pollution, v. 194, p. 121-129, nov. 2014. http://dx.doi.org/10.1016/j.envpol.2014.07.021

VIRK, G. et al. Microclimatic effects of green and cool roofs in London and their impacts on energy use for a typical office building. Energy and Buildings, v. 88, p. 214-228, Fevereiro 2015. https://doi.org/10.1016/j.enbuild.2014.11.039

WONG, I.; BALDWIN, A. N. Investigating the potential of applying vertical green walls to high-rise residential buildings for energy-saving in sub-tropical region. Building and Environment, v. 97, p. 34-39, 15 fev. 2016.

https://doi.org/10.1016/j.buildenv.2015.11.028

WONG, N. H. et al. Thermal evaluation of vertical greenery systems for building walls. Building and Environment, v. 45, n. 3, p. 663-672, mar. 2010. https://doi.org/10.1016/j.buildenv.2009.08.005

YANG, W. et al. Comparative study of the thermal performance of the novel green (planting) roofs against other existing roofs. Sustainable Cities and Society, v. 16, p. 1-12, Agosto 2015. http://dx.doi.org/10.1016/j.scs.2015.01.002

ZHAO, M. et al. Effects of plant and substrate selection on thermal performance of green roofs during the summer. Building and Environment, v. 78, p. 199-211, Agosto 2014. http://dx.doi.org/10.1016/j.buildenv.2014.02.011

\section{${ }^{1}$ Ketlin Bruna Montanari}

Arquiteta e Urbanista, mestranda do Programa de Pós Graduação em Arquitetura, Tecnologia e Cidade, UNICAMP. Endereço postal: Rua Saturnino de Brito, n 224, Cidade Universitária Zeferino Vaz, Campinas, SP, Brasil, CEP 13083-889

\section{${ }^{2}$ Lucila Chebel Labaki}

Física, Doutora em Ciências. Professora Titular no Departamento de Arquitetura e Construção, Faculdade de Engenharia Civil, Arquitetura e Urbanismo, UNICAMP. Endereço postal: Rua Saturnino de Brito, no 224, Cidade Universitária Zeferino Vaz, Campinas, SP, Brasil, CEP 13083-889 